\title{
Characterization of Mulberry Genetic Resources for Multiple Traits
}

\author{
M.C. Thriveni, Raju Mondal, G. Thanavendan, G. Ravikumar, B.T. Sreenivasa
}

\begin{abstract}
Mulberry plants have wide range of variations in characters in view of its adaptability to cross pollination with no inter-specific reproduction barrier rendering it a heterozygous species. Every plant being different from the other in natural population, this great diversity makes it difficult to gather information and carry out studies on effect of different edaphic factors on the expression of genotypic characters for adjudging the variations. In lieu of this known diversity for posterity, the collection of mulberry genotypes from diverse genetic sources, their conservation, evaluation and consequent documentation is of prime importance. In the present study, 69 mulberry accessions were characterized for morphology, anatomy, and reproductive parameters. Evaluation for propagation, growth and yield characters were also carried out. Based on multiple trait analysis, 14 top performing accessions viz., MI-0879, MI-0882, MI-0908, MI-0931, MI-0907, MI-0940, MI-0881, MI-0941, MI0892, MI-0913, MI-0937, MI-0934, MI-0865, MI-0886 were identified. These accessions could serve as resources for further evaluation aimed at trait-specific crop improvement.

Keywords: Characterization, Conservation, Mulberry (Morus sp.), Germplasm, Genetic resources
\end{abstract}

\section{INTRODUCTION}

Mulberry is a perennial, highly heterozygous, out breeding tree of the genus Morus within the family Moraceae. Morus species are widely distributed in Asia, Europe, North and South America. Mulberry leaf is the exclusive food for domestic silkworm Bombyx mori which produces natural silk. Hence, it has been regarded as the 'Queen of textiles'. However, the fine grain timber and tasty fruits of mulberry are also well known. Mulberry natural habitats in India are being explored for the collection and conservation. Central Sericultural Germplasm Resources Centre (CSGRC), Hosur is maintaining 1300 mulberry germplasm in the form of ex situ field gene bank.

Manuscript received on 01 July 2021 | Revised Manuscript received on 10 August 2021 | Manuscript Accepted on 15 October 2021 | Manuscript published on 30 October 2021. * Correspondence Author

M.C. Thriveni*, Central Sericultural Germplasm Resources Centre, Central Silk Board, Ministry of Textiles, Govt. of India, Thally road, P.B. No.44, Hosur (Tamil Nadu), India. Email: thrivenimc@gmail.com

Raju Mondal, Central Sericultural Germplasm Resources Centre, Central Silk Board, Ministry of Textiles, Govt. of India, Thally road, P.B. No. 44, Hosur- 635109 (Tamil Nadu), India.

G. Thanavendan, Central Sericultural Germplasm Resources Centre, Central Silk Board, Ministry of Textiles, Govt. of India, Thally road, P.B. No. 44, Hosur- 635109 (Tamil Nadu), India.

G. Ravikumar, Central Sericultural Germplasm Resources Centre, Central Silk Board, Ministry of Textiles, Govt. of India, Thally road, P.B. No. 44, Hosur- 635109 (Tamil Nadu), India.

B.T. Sreenivasa, Central Sericultural Germplasm Resources Centre, Central Silk Board, Ministry of Textiles, Govt. of India, Thally road, P.B. No. 44, Hosur- 635109 (Tamil Nadu), India.

(c) The Authors. Published by Lattice Science Publication (LSP). This is an open access article under the CC-BY-NC-ND license (http://creativecommons.org/licenses/by-nc-nd/4.0/)
Characterization of germplasm resources is one of the prerequisite for efficient utilization. Morus species exhibits rich phenotypic diversity. Many improved varieties are developed to meet the sericulture industry demand. The improvement in quantity and quality of mulberry genotypes over existing ones is a continuous process and breeders want suitable parental material for developing superior genotypes. Moreover, at present, there is an increasing demand to develop region and season specific mulberry varieties for rearing hybrid silkworm breeds. Standard characterization and evaluation of mulberry accessions are continuously carried out using different methods and traditional practices such as use of descriptor lists for morphology, anatomy, reproductive and growth characters. Characterization includes recording highly heritable phenotypic characters, which can be visually observed and expressed in various environments (Tikader and Kamble, 2010).

In order to identify the best performers and evolve new varieties suitable for specific zones, evaluation is essential. There is an increasing demand for new varieties suitable for various climatic conditions. To achieve this, identification of suitable parents from the germplasm accessions available is the need of the hour. In the present study, characterization of mulberry germplasm for different morphological, anatomical and reproductive characters was done. The germplasm was also evaluated for different growth and propagation traits. The trait-specific best performers were identified that can be utilized in future breeding programs.

\section{MATERIALS AND METHODS}

In the present study, 69 mulberry accessions were characterized for morphological, anatomical reproductive characters. They are also evaluated for propagation traits, growth and biochemical parameters. The data recorded was stored in the Mulberry Germplasm Information System (MGIS) database for further analysis.

\section{RESULTS}

\section{Morphological characterization of mulberry germplasm}

All the 69 mulberry germplasm accessions were characterized for 30 morphological descriptors as detailed in Table 1. Different morphological characters were recorded mainly by visual observations (branching pattern, colour of young and mature shoot, phyllotaxy, stipule nature, leaf colour, shape, texture, leaf apex and base, lobation type and margin). Among the accessions characterized, most of the accessions (47.83\%) were with spreading branching nature, 2/5 (37.68\%) phyllotaxy followed by $1 / 2$ (34.78\%), unlobed leaves (42.03\%) and homophyllus leaf nature (55\%).

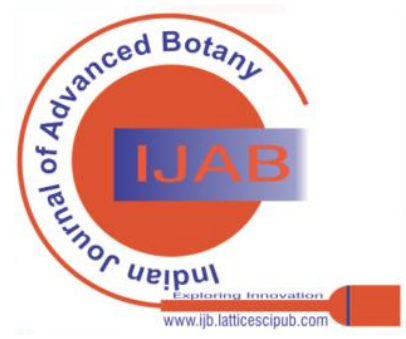


Leaf texture was mostly chartaceous (63.77\%), acuminate leaf apex (88.4\%), leaf margin serrate (59.42\%), cordate leaf base (72.46\%) and ovate leaf shape (71\%).

Table 1: Morphological characterization of mulberry

\begin{tabular}{|c|c|c|c|c|c|}
\hline \multicolumn{3}{|c|}{ Branching nature } & \multicolumn{3}{|c|}{ Leaf surface } \\
\hline Parameter & Frequency & $\%$ & Parameter & Frequency & $\%$ \\
\hline Erect & 11 & 15.94 & Rough & 25 & 36.23 \\
\hline Semi erect & 25 & 36.23 & Slightly rough & 13 & 18.84 \\
\hline Spreading & 33 & 47.83 & Smooth & 31 & 44.93 \\
\hline Total & 69 & 100.00 & Total & 69 & 100.00 \\
\hline \multicolumn{3}{|c|}{ Phyllotaxy } & \multicolumn{3}{|c|}{ Leaf texture } \\
\hline $1 / 2$ & 24 & 34.78 & Chartaceous & 44 & 63.77 \\
\hline $1 / 2,1 / 4$ & 1 & 1.45 & Coriaceous & 11 & 15.94 \\
\hline $1 / 2,2 / 5$ & 1 & 1.45 & Membranous & 14 & 20.29 \\
\hline $1 / 2,2 / 5$ & 1 & 1.45 & Total & 69 & 100.00 \\
\hline $1 / 3$ & 8 & 11.59 & \multicolumn{3}{|c|}{ Leaf apex } \\
\hline $1 / 3,2 / 5$ & 5 & 7.25 & Acuminate & 61 & 88.40 \\
\hline $2 / 5$ & 26 & 37.68 & Acute & 5 & 7.25 \\
\hline $2 / 5,1 / 3$ & 3 & 4.35 & Caudate & 3 & 4.35 \\
\hline Total & 69 & 100.00 & Total & 69 & 100.00 \\
\hline \multicolumn{3}{|c|}{ Leaf lobation type } & \multicolumn{3}{|c|}{ Leaf margin } \\
\hline Deep lobed & 3 & 4.35 & Crenate & 10 & 14.49 \\
\hline Medium lobed & 24 & 34.78 & Dentate & 18 & 26.09 \\
\hline Shallow lobed & 13 & 18.84 & Serrate & 41 & 59.42 \\
\hline Unlobed & 29 & 42.03 & \multirow[t]{2}{*}{ Total } & \multirow[t]{2}{*}{69} & \multirow[t]{2}{*}{100.00} \\
\hline Total & 69 & 100.00 & & & \\
\hline
\end{tabular}

\section{Anatomical characterization of mulberry germplasm}

In order to study leaf anatomical characters, fully expanded leaves from $7^{\text {th }}$ to $9^{\text {th }}$ position from top of the longest shoot in descending order were collected in morning hours between 9 and 10 AM from 3 month old shoots. The stomata and idioblasts were studied by taking a thin layer of Wimbley's quick fix impressions of the abaxial (lower) and adaxial (upper) leaf surface and observed under Leica microscope. Stomata and idioblasts were counted per unit area and the frequency per $\mathrm{mm}^{2}$ was calculated.

Studies on the cuticle, epidermal, palisade and spongy mesophyll, leaf thickness and idioblast length and breadth were carried out by making thin hand sections using steel razor blade (super max) that were observed under the Leica microscope. The chloroplast number per stomata was counted using epidermal peelings of freshly collected leaf samples stained with $2 \%$ potassium iodide-iodine solution observed under the microscope. The data was subjected for statistical analysis viz. mean, maximum, minimum, standard error (SE) and co-efficient of variation ( $\mathrm{CV} \%)$ for completely randomized design to determine the significance of treatment (Table 2). The top performing ten accessions for different anatomical traits and range of variation for these accessions are presented in the Table 3.

Table 2: Anatomical characterization of mulberry germplasm

\begin{tabular}{|l|c|c|c|c|c|}
\hline \multicolumn{1}{|c|}{ Parameters } & Mean & Min. & Max. & SE & CV\% \\
\hline Stomatal size $($ sq. $\mu \mathrm{m})$ & 244.71 & 176.76 & 341.45 & 4.36 & 14.69 \\
\hline Stomatal frequency $($ no./sq.mm) & 796.16 & 320.29 & 1728.32 & 38.59 & 39.97 \\
\hline Idioblast length $(\mu \mathrm{m})$ & 29.78 & 18.28 & 70.69 & 1.20 & 33.11 \\
\hline Idioblast width $(\mu \mathrm{m})$ & 33.91 & 21.72 & 64.18 & 0.95 & 23.04 \\
\hline Idioblast frequency no./sq.mm) & 18.92 & 8.63 & 34.54 & 0.65 & 28.33 \\
\hline Palisade thickness $(\mu \mathrm{m})$ & 61.72 & 35.86 & 103.45 & 1.72 & 22.99 \\
\hline Spongy thickness $(\mu \mathrm{m})$ & 65.23 & 35.17 & 103.45 & 1.50 & 18.98 \\
\hline Palisade-spongy ratio & 0.94 & 0.71 & 1.46 & 0.02 & 13.81 \\
\hline Upper cuticle thickness $(\mu \mathrm{m})$ & 7.75 & 3.45 & 12.74 & 0.22 & 22.73 \\
\hline Lower cuticle thickness $(\mu \mathrm{m})$ & 4.26 & 2.93 & 6.99 & 0.14 & 27.29 \\
\hline Upper epidermis thickness $(\mu \mathrm{m})$ & 23.07 & 14.18 & 41.38 & 0.67 & 23.97 \\
\hline Lower epidermis thickness $(\mu \mathrm{m})$ & 9.43 & 3.45 & 16.48 & 0.32 & 27.56 \\
\hline Leaf thickness $(\mu \mathrm{m})$ & 171.28 & 111.72 & 272.41 & 3.25 & 15.66 \\
\hline No. of chloroplast/stomata $(\mathrm{no})$. & 11.98 & 8.67 & 19.11 & 0.35 & 19.51 \\
\hline
\end{tabular}

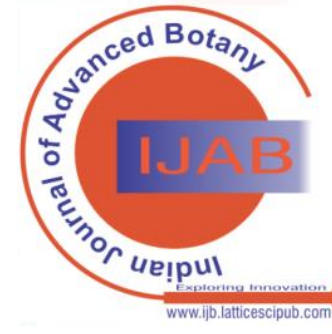


Table 3: Top performing accessions for different anatomical characters

\begin{tabular}{|c|c|c|}
\hline Parameters & Range & Top performing accessions \\
\hline Stomatal size (sq. $\mu \mathrm{m})$ & $176.76-204.30$ & $\begin{array}{l}\text { MI-0866, MI-0942, MI-0931, MI-0867, MI-0932, MI- } \\
\text { 0913, MI-0964, MI-0927, MI-0908, MI-0928 }\end{array}$ \\
\hline $\begin{array}{l}\text { Stomatal frequency } \\
\text { (no./sq.mm) }\end{array}$ & $320.29-476.50$ & $\begin{array}{l}\text { MI-0883, MI-0886, MI-0882, MI-0887, MI-0889, MI- } \\
\text { 0879, MI-0884, MI-0892, MI-0873, MI-0868 }\end{array}$ \\
\hline Palisade thickness $(\mu \mathrm{m})$ & 73.791-103.45 & $\begin{array}{l}\text { MI-0926, MI-0892, MI-0946, MI-0867, MI-0884, MI- } \\
\text { 0951, MI-0870, MI-0911, MI-0879, MI-0906 }\end{array}$ \\
\hline Palisade-spongy ratio & $1.062-1.46$ & $\begin{array}{l}\text { MI-0892, MI-0911, MI-0864, MI-0873, MI-0870, MI- } \\
\text { 0913, MI-0883, MI-0914, MI-0881, MI-0946 }\end{array}$ \\
\hline $\begin{array}{l}\text { Upper cuticle thickness } \\
(\mu \mathrm{m})\end{array}$ & $9.66-12.74$ & $\begin{array}{l}\text { MI-0884, MI-0947, MI-0866, MI-0930, MI-0939, MI- } \\
\text { 0868, MI-0881, MI-0916, MI-0963, MI-0917 }\end{array}$ \\
\hline $\begin{array}{l}\text { Lower cuticle thickness } \\
(\mu \mathrm{m})\end{array}$ & $5.52-6.99$ & $\begin{array}{l}\text { MI-0872, MI-0919, MI-0904, MI-0923, MI-0926, MI- } \\
\text { 0928, MI-0907, MI-0920, MI-0874, MI-0959 }\end{array}$ \\
\hline Leaf thickness $(\mu \mathrm{m})$ & $195.69-272.41$ & $\begin{array}{l}\text { MI-0926, MI-0867, MI-0870, MI-0951, MI-0872, MI- } \\
\text { 0884, MI-0879, MI-0946, MI-0892, MI-0928 }\end{array}$ \\
\hline No. of chloroplasts/ stomata & $13.4-19.11$ & $\begin{array}{l}\text { MI-0889, MI-0959, MI-0963, MI-0912, MI-0870, MI- } \\
\text { 0885, MI-0872, MI-0887, MI-0886, MI-0917 }\end{array}$ \\
\hline
\end{tabular}

\section{Reproductive characterization of mulberry germplasm}

The reproductive characterization was recorded in the main flowering season (February- March). Fully bloomed catkins of different accessions were collected and the length as well as breadth of pedicel was measured. The number of flowers/ catkins of different sexes were counted by dissecting the individual flowers around the rachis of the inflorescence. Length of style and stigma, nature and type of the female flowers were recorded by dissecting them under the stereomicroscope at receptive stage. The male catkins were collected in plastic bags at $10 \mathrm{AM}$ at the time of anthesis and the length of stamen and anther were measured under stereomicroscope. To study the pollen diameter, viability and number of pollen germ pores, the mature pollen grains were dusted on a clean slide and stained with $2 \%$ acetocarmine solution for 30 minutes. After 30 minutes, the slides were observed under microscope. Fruit characters were studied using fully ripened sorosis and recording of the length, breadth, pedicle length, weight, colour and taste of the fruit. The data was subjected to statistical analysis like mean, maximum, minimum, standard error (SE) and coefficient of variation ( $\mathrm{CV} \%$ ) to determine the significance of treatment (Table 4).

Top performing accessions for different anatomical traits and range of variation for these accessions are presented in table 5 .

Table 4: Reproductive characterization of mulberry germplasm

\begin{tabular}{|l|c|c|c|c|c|}
\hline \multicolumn{1}{|c|}{ Parameters } & Mean & Min. & Max. & SE & CV\% \\
\hline Male inflorescence length (cm) & 2.29 & 0.86 & 8.54 & 0.30 & 63.45 \\
\hline Female inflorescence length (cm) & 1.33 & 0.42 & 4.98 & 0.13 & 71.74 \\
\hline Bisexual inflorescence length (cm) & 1.42 & 0.62 & 2.10 & 0.20 & 35.14 \\
\hline Male inflorescence diameter (cm) & 0.53 & 0.34 & 0.78 & 0.02 & 17.21 \\
\hline Female inflorescence diameter (cm) & 0.52 & 0.38 & 0.80 & 0.01 & 15.14 \\
\hline Bisexual inflorescence diameter (cm) & 0.48 & 0.38 & 0.56 & 0.03 & 15.77 \\
\hline No. of flowers/catkin (male) & 33.29 & 16.00 & 147.60 & 5.02 & 73.90 \\
\hline No. of flowers/catkin (female) & 37.81 & 9.00 & 152.00 & 3.40 & 67.35 \\
\hline No. of flowers/catkin (bisexual) & 25.24 & 14.00 & 54.60 & 4.78 & 50.05 \\
\hline Male peduncle length (cm) & 0.60 & 0.26 & 1.20 & 0.04 & 36.63 \\
\hline Female peduncle length (cm) & 0.69 & 0.18 & 1.76 & 0.04 & 43.43 \\
\hline Bisexual peduncle length (cm) & 0.50 & 0.26 & 0.80 & 0.08 & 38.59 \\
\hline Stamen length (mm) & 3.32 & 1.54 & 4.90 & 0.21 & 30.94 \\
\hline Anther length (cm) & 1.13 & 0.44 & 2.78 & 0.10 & 42.29 \\
\hline Pollen diameter $(\mu m)$ & 17.97 & 0.62 & 26.80 & 0.90 & 24.91 \\
\hline Pollen viability (\%) & 96.43 & 1.60 & 99.00 & 8.32 & 43.17 \\
\hline
\end{tabular}




\begin{tabular}{|l|l|l|l|l|l|}
\hline Style length (mm) & 0.62 & 0.12 & 1.76 & 0.05 & 60.02 \\
Stigma length (mm) & 4.93 & 1.40 & 8.58 & 0.20 & 31.39 \\
\hline Fruit length $(\mathrm{cm})$ & 1.97 & 0.94 & 4.98 & 0.13 & 46.67 \\
\hline Fruit width $(\mathrm{cm})$ & 0.84 & 0.24 & 1.24 & 0.03 & 23.40 \\
\hline Fruit weight $(\mathrm{g})$ & 0.95 & 0.18 & 2.81 & 0.08 & 62.42 \\
\hline
\end{tabular}

Table 5: Top performing accessions for different reproductive characters

\begin{tabular}{|l|c|l|}
\hline \multicolumn{1}{|c|}{ Parameters } & Range & \multicolumn{1}{c|}{ Top performing accessions } \\
\hline Male inflorescence length (cm) & $1.54-1.56$ & MI-0815, MI-0860 \\
\hline Male inflorescence diameter (cm) & $0.54-0.58$ & MI-0980, MI-0815 \\
\hline No. of flowers/ catkin (male) & $34.2-49.2$ & MI-0980, MI-0860 \\
\hline Male peduncle length (cm) & $0.36-0.5$ & MI-0860, MI-0980 \\
\hline Style length (cm) & $0.44-2.9$ & $\begin{array}{l}\text { MI-0974, MI-0975, MI-0970, MI-0973, } \\
\text { MI-0900, MI-0877, MI-0962, MI-0878, } \\
\text { MI-0869, MI-0857 }\end{array}$ \\
\hline Stigma length (cm) & $3.06-8.84$ & $\begin{array}{l}\text { MI-0973, MI-0974, MI-0975, MI-0878, } \\
\text { MI-0900, MI-0857, MI-0970, MI-0877, } \\
\text { MI-0838, MI-0869 }\end{array}$ \\
\hline Fruit length (cm) & $1.8-3.74$ & $\begin{array}{l}\text { MI-0878, MI-0857, MI-0962, MI-0814, } \\
\text { MI-0974, MI-0975, MI-0966, MI-0900, } \\
\text { MI-0816, MI-0973 }\end{array}$ \\
\hline Fruit diameter (cm) & & $\begin{array}{l}\text { MI-0962, MI-0839, MI-0966, MI-0973, } \\
\text { MI-0974, MI-0838, MI-0975, MI-0814, } \\
\text { MI-0837, MI-0900 }\end{array}$ \\
\hline Fruit peduncle (cm) & $0.86-1.28$ & $\begin{array}{l}\text { MI-0966, MI-0859, MI-0970, MI-0962, } \\
\text { MI-0816, MI-0877, MI-0814, MI-0838, } \\
\text { MI-0837, MI-0975 }\end{array}$ \\
\hline Fruit weight (g) & $0.2-0.44$ & $\begin{array}{l}\text { MI-0962, MI-0966, MI-0839, MI-0814, } \\
\text { MI-0878, MI-0973, MI-0837, MI-0975, } \\
\text { MI-0974, MI-0816 }\end{array}$ \\
\hline
\end{tabular}

\section{Evaluation of mulberry germplasm for growth and yield parameters}

After 70 days of pruning the growth and yield traits were recorded. The total number of shoots/ plant was harvested from the basal node and the number of primary shoots/ plant was counted considering four plants for each accession. The longest shoot which attained maximum growth in the plant was considered for assessing the length of longest shoot. The intermodal distance and lengths of 10 internodes on the longest shoot leaving $1 / 3^{\text {rd }}$ top portion were measured. The largest leaf between $7^{\text {th }}$ to $9^{\text {th }}$ positions from the top was considered for measuring lamina length and weighing lamina weight. Total aerial biomass of the plant was determined considering shoot weight along with the foliage; the entire foliage was harvested and total leaf yield was calculated. Leaves were collected from the longest shoot and fresh weight was recorded. Dry weight was recorded leaves dried in the oven at $60^{\circ} \mathrm{C}$ for $48 \mathrm{~h}$. The moisture content of the excised leaves was calculated using the formula:

\section{Moisture content $(\mathrm{MC})=\underline{\text { (Fresh weight }- \text { oven dry weight }) \times 100}$ \\ Fresh weight}

The leaf moisture content after $6 \mathrm{~h}$ was measured taking fresh weight of the leaves immediately after collecting from the longest shoot and then after $6 \mathrm{~h}$. The leaf dry weight was recorded after drying the leaf samples in oven for $48 \mathrm{~h}$ at $60^{\circ}$ C. The moisture retention capacity (MRC) was calculated as using the following formula:

\section{Moisture retention capacity $(\mathrm{MRC})=(6 \mathrm{~h}$ weight - oven dry weight $) \times 100$ Fresh weight - dry weight}

Statistical analysis such as mean, maximum, minimum, standard error (SE) and co-efficient of variation (CV \%) was conducted to determine the significance of treatment (Table
6). Among the accessions characterized, top performing accessions for different propagation traits and range of variation for these accessions are presented in the Table 7.

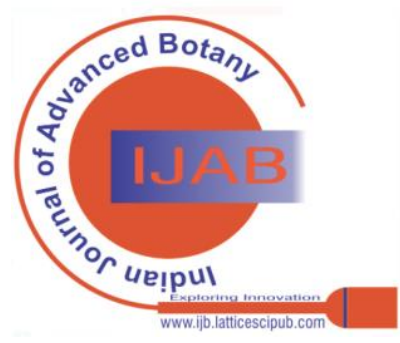


Table 6: Evaluation of mulberry germplasm for growth and yield parameters

\begin{tabular}{|l|c|c|c|c|}
\hline \multicolumn{1}{|c|}{ Parameter } & Mean & Range & SE & CV\% \\
\hline No. of branches & 29.8 & $8-72$ & 1.69 & 46.64 \\
\hline Length of the longest shoot (cm) & 148.47 & $74-258$ & 4.45 & 24.72 \\
\hline Inter nodal distance (cm) & 5.13 & $1.9-10.56$ & 0.19 & 31.27 \\
\hline Wt. of single leaf (g) & 3.73 & $0.75-10.35$ & 0.27 & 60.58 \\
\hline Leaf yield/plant (kg) & 1.61 & $0.30-4.84$ & 0.11 & 58.61 \\
\hline Biomass/plant (kg) & 2.98 & $0.45-11.58$ & 0.24 & 65.30 \\
\hline Leaf Moisture Content \% & 71.23 & $65.89-79.45$ & 0.29 & 3.39 \\
\hline MC \% after 6 hours & 63.22 & $45.42-72.09$ & 0.68 & 8.91 \\
\hline
\end{tabular}

Table 7: Better performing accessions for different growth and yield parameters

\begin{tabular}{|c|c|c|}
\hline Parameter & Range & Better performing accessions \\
\hline No. of branches & $72.5-44.5$ & $\begin{array}{l}\text { MI-0928, MI-0938, MI-0872, MI-0958, } \\
\text { MI-0927, MI-0907, MI-0881, MI-0931, } \\
\text { MI-0917, MI-0913 }\end{array}$ \\
\hline $\begin{array}{l}\text { Length of the longest } \\
\text { shoot }(\mathrm{cm})\end{array}$ & $258-186$ & $\begin{array}{l}\text { MI-0883, MI-0908, MI-0907, MI-0886, } \\
\text { MI-0879, MI-0918, MI-0919, MI-0889, } \\
\text { MI-0872, MI-0880 }\end{array}$ \\
\hline Inter-nodal distance (cm) & $1.9-3.77$ & $\begin{array}{l}\text { MI-0926, MI-0914, MI-0931, MI-0906, } \\
\text { MI-0959, MI-0912, MI-0875, MI-0913, } \\
\text { MI-0964, MI-0917 }\end{array}$ \\
\hline Wt. of single leaf (g) & $\begin{array}{c}10.35- \\
6.17\end{array}$ & $\begin{array}{l}\text { MI-0887, MI-0883, MI-0907, MI-0963, } \\
\text { MI-0925, MI-0941, MI-0885, MI-0934, } \\
\text { MI-0960, MI-0944 }\end{array}$ \\
\hline Total leaf yield (kg) & $4.84-2.74$ & $\begin{array}{l}\text { MI-0907, MI-0882, MI-0938, MI-0925, } \\
\text { MI-0928, MI-0872, MI-0923, MI-0908, } \\
\text { MI-0922, MI-0936 }\end{array}$ \\
\hline Biomass (kg) & $\begin{array}{c}11.58- \\
5.08\end{array}$ & $\begin{array}{l}\text { MI-0907, MI-0908, MI-0882, MI-0928, } \\
\text { MI-0938, MI-0922, MI-0872, MI-0925, } \\
\text { MI-0887, MI-0936 }\end{array}$ \\
\hline Moisture Content (\%) & $\begin{array}{l}79.45- \\
73.57\end{array}$ & $\begin{array}{l}\text { MI-0922, MI-0884, MI-0904, MI-0885, } \\
\text { MI-0889, MI-0886, MI-0908, MI-0864, } \\
\text { MI-0882, MI-0868 }\end{array}$ \\
\hline MC \% after 6 hours & $\begin{array}{l}72.09- \\
68.65\end{array}$ & $\begin{array}{l}\text { MI-0922, MI-0885, MI-0908, MI-0904, } \\
\text { MI-0879, MI-0884, MI-0889, MI-0870, } \\
\text { MI-0864, MI-0868 }\end{array}$ \\
\hline
\end{tabular}

Evaluation of mulberry germplasm for propagation traits

Mulberry is generally vegetatively propagated through stem cuttings. To understand the genetic potential of the mulberry accessions, propagation traits evaluated. The rooting ability and root characters were recorded which help in proper establishment of the plant. To evaluate mulberry accessions for propagation traits, 6 months old mulberry shoots are used to prepare cuttings with 3-4 buds and planted in the nursery in RBD in 3 replications along with the checks. 20 cuttings per replication were planted at a spacing of $10 \mathrm{~cm}$ between the cuttings and $20 \mathrm{~cm}$ between the rows. The evaluations are carried out after 90 days of planting when the saplings are uprooted carefully without damaging the roots for recording observations on roots. The statistically analyzed data is given in Table 8. Among the evaluated accessions, 10 top performing accessions for each character were identified as given in Table 9.

Table 8: Evaluation of mulberry germplasm for propagation traits

\begin{tabular}{|l|c|c|c|c|c|}
\hline \multicolumn{1}{|c|}{ Parameter } & Mean & Min. & Max. & SE & CV\% \\
\hline Rooting ability (\%) & 79.63 & 0.00 & 95 & 2.76 & 25.23 \\
\hline No. of roots/sapling & 4.64 & 1.17 & 8.83 & 0.29 & 46.03 \\
\hline Length of longest root & 25.55 & 5.5 & 48.5 & 1.36 & 38.77 \\
\hline Dry root weight (g) & 1.37 & 0.05 & 6.63 & 0.17 & 89.63 \\
\hline Root Volume & 3.08 & 0.17 & 14.5 & 0.37 & 86.58 \\
\hline
\end{tabular}




\begin{tabular}{|l|l|l|l|l|l|}
\hline Root shoot Ratio by dwt & 0.34 & 0.06 & 0.85 & 0.02 & 43.10 \\
\hline Root shoot Ratio by length & 0.90 & 0.25 & 1.76 & 0.05 & 37.19 \\
\hline
\end{tabular}

Table 9: Better performing accessions for different propagation traits

\begin{tabular}{|l|c|l|}
\hline \multicolumn{1}{|c|}{ Parameter } & Range & \multicolumn{1}{|c|}{ Better performing accessions } \\
\hline Rooting ability (\%) & $95-95$ & $\begin{array}{l}\text { MI-0938, MI-0922, MI-0882, MI-0928, MI-0893, } \\
\text { MI-0864, MI-0916, MI-0934, MI-0913, MI-0921 }\end{array}$ \\
\hline No. of roots/sapling & $8.83-6.83$ & $\begin{array}{l}\text { MI-0940, MI-0907, MI-0882, MI-0942, MI-0964, } \\
\text { MI-0913, MI-0871, MI-0875, MI-0935, MI-0916 }\end{array}$ \\
\hline Length of longest root & $48.5-34.67$ & $\begin{array}{l}\text { MI-0916, MI-0879, MI-0940, MI-0906, MI-0933, } \\
\text { MI-0873, MI-0941, MI-0931, MI-0886, MI-0908 }\end{array}$ \\
\hline Dry root weight (g) & $6.63-2.28$ & $\begin{array}{l}\text { MI-0940, MI-0875, MI-0964, MI-0916, MI-0941, } \\
\text { MI-0935, MI-0931, MI-0937, MI-0873, MI-0934 }\end{array}$ \\
\hline Root Volume & $14.5-5.33$ & $\begin{array}{l}\text { MI-0940, MI-0875, MI-0935, MI-0964, MI-0941, } \\
\text { MI-0937, MI-0942, MI-0931, MI-0916, MI-0882 }\end{array}$ \\
\hline Root shoot Ratio by weight & $0.85-0.44$ & $\begin{array}{l}\text { MI-0959, MI-0865, MI-0932, MI-0935, MI-0943, } \\
\text { MI-0906, MI-0870, MI-0963, MI-0941, MI-0950 }\end{array}$ \\
\hline Root shoot Ratio by length & $1.76-1.18$ & $\begin{array}{l}\text { MI-0920, MI-0933, MI-0921, MI-0950, MI-0881, } \\
\text { MI-0870, MI-0959, MI-0914, MI-0941, MI-0943 }\end{array}$ \\
\hline
\end{tabular}

\section{Evaluation of mulberry germplasm for biochemical} parameters

With respect to biochemical characterization, the leaf chlorophyll content from fresh leaves, and water soluble protein and carbohydrates from dry leaf powders were studied. The amount of chlorophyll present in the leaf extract was calculated using the formula:

Chlorophyll a (mg/ g F.W.) $=\left(12.7 A_{663}-2.69 A_{645}\right) \times$ v/ $1000 \times$ wt.

Chlorophyll b (mg/g F.W.) $=(22.9$ A645 - 4.68 A663) x V/ 1000 x wt.
Total Chlorophyll (mg/g F.W. $)=\left(20.2 A_{645}+8.02 A_{663}\right) x$ V/ 1000 x wt.

where,

$\mathrm{A}_{645}=$ Absorption value at $645 \mathrm{~nm}, \mathrm{~A}_{663}=$ absorption value at $663 \mathrm{~nm}$

DMSO)

$$
\mathrm{V}=\text { total volume of chlorophyll extract (i.e., }
$$

Wt. = weight of the leaf tissue

The statistically analyzed data is given in Table 10. Among the evaluated accessions, 10 top performing accessions for each character were identified as given in Table 11.

Table 10: Evaluation of mulberry germplasm for biochemical parameters

\begin{tabular}{|l|c|c|c|c|c|}
\hline \multicolumn{1}{|c|}{ Parameter } & Mean & Min. & Max. & SE & CV\% \\
\hline Chlorophyll a (mg/g f.wt.) & 1.711 & 1.035 & 2.298 & 0.037 & 17.65 \\
\hline Chlorophyll b (mg/g f.wt.) & 0.308 & 0.149 & 0.786 & 0.013 & 33.90 \\
\hline Total Chlorophyll (mg/g f.wt.) & 2.013 & 1.198 & 2.889 & 0.047 & 19.20 \\
\hline Chlorophyll a/b & 5.918 & 2.681 & 8.49 & 0.136 & 18.99 \\
\hline Protein soluble (\% f.wt.) & 2.169 & 1.083 & 3.64 & 0.067 & 25.61 \\
\hline Protein soluble (\% d.wt.) & 7.719 & 4.060 & 12.81 & 0.225 & 24.08 \\
\hline Carbohydrate soluble (\% f.wt.) & 3.742 & 1.293 & 6.78 & 0.168 & 37.02 \\
\hline Carbohydrate soluble (\% d.wt.) & 12.921 & 4.617 & 22.53 & 0.536 & 34.19 \\
\hline
\end{tabular}


Table 11: Better performing accessions for different biochemical parameters

\begin{tabular}{|l|c|l|}
\hline \multicolumn{1}{|c|}{ Parameter } & Range & \multicolumn{1}{c|}{ Better performing accessions } \\
\hline Chlorophyll a (mg/g f.wt.) & $2.30-2.06$ & $\begin{array}{l}\text { MI-0886, MI-0882, MI-0884, MI-0964, MI-0908, } \\
\text { MI-0931, MI-0892, MI-0887, MI-0885, MI-0883 }\end{array}$ \\
\hline Chlorophyll b (mg/g f.wt.) & $0.41-0.79$ & $\begin{array}{l}\text { MI-0887, MI-0885, MI-0883, MI-0870, MI-0908, } \\
\text { MI-0886, MI-0881, MI-0882, MI-0922, MI-0889 }\end{array}$ \\
\hline Total Chlorophyll (mg/g f.wt) & $2.47-2.89$ & $\begin{array}{l}\text { MI-0887, MI-0886, MI-0882, MI-0884, MI-0908, } \\
\text { MI-0964, MI-0885, MI-0883, MI-0892, MI-0931 }\end{array}$ \\
\hline Chlorophyll a/b & $7.13-8.49$ & $\begin{array}{l}\text { MI-0929, MI-0911, MI-0936, MI-0934, MI-0952, } \\
\text { MI-0932, MI-0912, MI-0938, MI-0917, MI-0959 }\end{array}$ \\
\hline $\begin{array}{l}\text { Protein soluble } \\
\text { (\% f.wt.) }\end{array}$ & $2.72-3.64$ & $\begin{array}{l}\text { MI-0936, MI-0932, MI-0867, MI-0871, MI-0959, } \\
\text { MI-0951, MI-0872, MI-0950, MI-0864, MI-0921 }\end{array}$ \\
\hline Protein soluble (\% d.wt.) & $9.66-12.81$ & $\begin{array}{l}\text { MI-0937, MI-0867, MI-0871, MI-0864, MI-0933, } \\
\text { MI-0872, MI-0904, MI-0916, MI-0868, MI-0960 }\end{array}$ \\
\hline Carbohydrate soluble (\% f.wt.) & $5.31-6.78$ & $\begin{array}{l}\text { MI-0916, MI-0951, MI-0914, MI-0913, MI-0960, } \\
\text { MI-0912, MI-0949, MI-0958, MI-0904, MI-0950 }\end{array}$ \\
\hline Carbohydrate soluble (\% d.wt.) & $17.04-22.53$ & $\begin{array}{l}\text { MI-0916, MI-0914, MI-0904, MI-0913, MI-0960, } \\
\text { MI-0912, MI-0951, MI-0963, MI-0937, MI-0949 }\end{array}$ \\
\hline
\end{tabular}

The 69 accessions studied were subjected to multiple trait analysis (anatomical, growth, yield, biochemical and propagation traits) resulting in the identification of 14 promising accessions viz., MI-0879, MI-0882, MI-0908,
MI-0931, MI-0907, MI-0940, MI-0881, MI-0941, MI-0892, MI-0913, MI-0937, MI-0934, MI-0865, MI-0886 (Table 12).

Table 12: Top performing accessions based on multiple traits

\begin{tabular}{|c|c|c|}
\hline Acc.No. & No. of traits & Trait No. (value) \\
\hline MI-0879 & 18 & $\begin{array}{l}\text { 1(33), 2(195.333), 4(4.787), 5(2.736), 6(4.64), 7(229.144), 8(446.582), } \\
\text { 9(78.925), 10(1.044), 11(9.195), 12(206.03), 13(85), 15(45), 17(3.67), 19(0.97), } \\
\text { 20(2.153), 23(73.03), 24(69.82), }\end{array}$ \\
\hline MI-0882 & 18 & 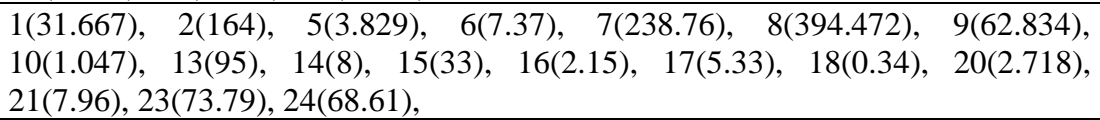 \\
\hline MI-0908 & 16 & $\begin{array}{l}\text { 2(234), 4(6.017), 5(3.052), 6(7.42), 7(204.072), 9(64.826), 10(1.046), 14(5), } \\
\text { 15(35), 16(1.463), 17(3.27), 18(0.38), 19(1.04), 20(2.683), 23(74.27), 24(70.54), }\end{array}$ \\
\hline MI-0931 & 16 & $\begin{array}{l}\text { 1(47), 2(162), 3(33), 5(1.662), 6(3.51), 7(183.183), 8(605.226), 11(8.276), } \\
\text { 13(85), 14(5), 15(36), 16(2.863), 17(5.73), 18(0.43), 20(2.468), 22(13.427), }\end{array}$ \\
\hline MI-0907 & 16 & $\begin{array}{l}\text { 1(50.5), 2(215.5), 4(8.987), 5(4.841), 6(11.58), 9(70.343), 10(0.996), } \\
\text { 12(179.306), 13(95), 14(9), 15(26), 19(1.12), 20(2.332), 21(8.887), 22(16.377), } \\
\text { 24(66.11), }\end{array}$ \\
\hline MI-0940 & 16 & $\begin{array}{l}\text { 1(36), 3(23), 4(4.017), 5(2.323), 6(4.64), 7(227.258), 8(682.179), 13(85), 14(9), } \\
\text { 15(39), 16(6.632), 17(14.5), 18(0.34), 20(2.374), 21(9.07), 22(14.217), }\end{array}$ \\
\hline MI-0881 & 16 & $\begin{array}{l}\text { 1(47.333), 2(152.667), 3(23), 5(1.779), 6(3.12), 8(497.422), 9(69.73), 10(1.063), } \\
\text { 11(9.961), 12(184.67), 13(80), 15(26), 19(1.47), 20(2.276), 23(73.43), 24(67.54), }\end{array}$ \\
\hline MI-0941 & 16 & 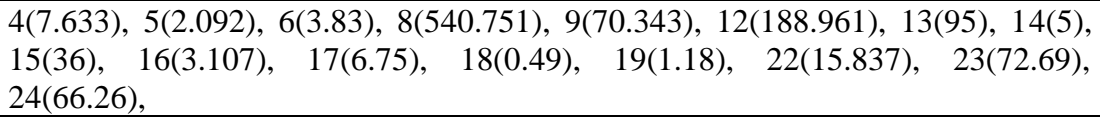 \\
\hline MI-0892 & 15 & $\begin{array}{l}\text { 2(170), 3(26), 5(1.969), 6(3.26), 8(464.838), 9(97.929), 10(1.456), 12(198.616), } \\
\text { 13(95), 14(6), 18(0.41), 20(2.539), 21(9.19), 23(73.19), 24(66.28), }\end{array}$ \\
\hline MI-0913 & 15 & $\begin{array}{l}\text { 1(44.5), 3(28), 7(194.202), 8(617.704), 9(64.826), 10(1.093), 11(9.655), } \\
\text { 12(175.858), 13(95), 14(8), 15(33), 16(1.482), 17(3.1), 19(1.02), 22(21.13), }\end{array}$ \\
\hline MI-0937 & 15 & $\begin{array}{l}\text { 4(5.78), 5(2.183), 6(3.79), 8(542.831), 13(90), 14(5), 15(30), 16(2.75), 17(6.25), } \\
\text { 19(1.02), 20(2.267), 21(12.81), 22(17.687), 23(72.87), 24(66.56), }\end{array}$ \\
\hline MI-0934 & 14 & 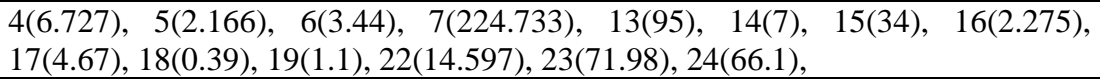 \\
\hline MI-0865 & 14 & $\begin{array}{l}\text { 1(43), 3(24), 7(221.289), 8(538.672), 10(0.988), 11(8.965), 13(95), 18(0.69), } \\
\text { 19(0.99), 20(2.167), 21(8.05), 22(13.86), 23(71.95), 24(65.56), }\end{array}$ \\
\hline MI-0886 & 14 & $\begin{array}{l}\text { 2(200), 5(1.753), 6(3.74), 8(385.921), 9(70.88), 12(188.214), 13(95), 15(36), } \\
\text { 17(3.33), 19(1.06), 20(2.749), 21(8.053), 23(74.53), 24(67.69), }\end{array}$ \\
\hline
\end{tabular}




\section{DISCUSSION}

In the present investigation, 69 mulberry germplasm were characterized for different morphological, anatomical, reproductive, biochemical, growth and yield characters. The basis of conservation is availability of sufficient knowledge on species diversity and the ecosystem in which they are present. Ex situ conservation is the practical way of preservation to date for storage of mulberry germplasm i.e., in a gene bank which usually means a seed bank. However, in case of mulberry, seed storage is difficult or almost impossible as the genetic integrity of a particular clone or genotype cannot be preserved due to high degree of heterozygosity. In mulberry, the inherited characteristics of the parental stocks can be perpetuated only by vegetative propagation (Bindroo et al., 2012).

Peris et al. (2014) studied five mulberry accessions for genetic divergence using 12 phenotypic traits in two localities, Nairobi and Eldoret. Their results indicated that the tested accessions were clustered in four groups. The traits tested across the five accessions can be utilized for future breeding for high yielding varieties. Chatterjee et al. (2004) have studied morphological and molecular variation in Morus laevigata collected from wide agro-climatic zones using 12 morpho-biochemical parameters and RAPD profiles. The study established significant variability for only two morpho-biochemical parameters (100 leaf weight and stomata number). Jayeoba et al. (2014) has studied the morphological and chemical analysis of eight varieties of Morus alba to determine the differences among them. The results revealed that K2 and S34 were significant varieties.

\section{CONCLUSION}

In the present study, it is evident that, effective and efficient utilization of germplasm depends on the detailed and systematic characterization. Phenotypic characterization is essential in the description and classification of the germplasm. In addition, it facilitates in addressing the trueness and detecting the duplicates. However, the present work needs to be complemented with molecular studies to strengthen the morphological differences.

\section{ACKNOWLEDGEMENTS}

The authors are thankful to Central Sericultural Germplasm Resources Centre, Central Silk Board, Ministry of Textiles, Govt. of India for providing the facilities and financial assistance. Dr. K. Jhansilakshmi, Scientist-D is duly acknowledged for data collection and interpretation.

\section{REFERENCES}

1. Tikader A and Kamble C.K. (2008). Studies on variability of indigenous mulberry germplasm on growth and leaf yield. Pertanika Journal of Tropical Agricultural Science 31 (2): 163-170.

2. Tikader A and Kamble C.K. (2010). Development of promising mulberry germplasm using wild genes. Indian Silk 48 (11): 12-13.

3. Bindroo BB, Chowdhuri SR and Ghosh MK (2012). Diversity, distribution and conservation of mulberry (Morus sp.) in Himalyas. Journal of Crop and Weed 8 (1): 26-30.

4. Peris NW, Gacheri KM, Theophillus MM and Lucas N (2014). Morphological characterization of Mulberry (Morus spp.) accessions grown in Kenya. Sustainable Agriculture Research 3 (1): 10-17. [CrossRef]

5. Chatterjee SN, Nagaraja GM, Srivastava PP and Naik G (2004). Morphological and molecular variation of Morus laevigata in India. Genetica, 121: 133-143. [CrossRef]
6. Jayeoba FM, Ogunbanjo OR and Awotoye OO (2014) Characterization of mulberry (Morus alba L.) varieties in South Western Nigeria. 6. Continental Journal of Agricultural Science 8 (1) 13-19.

\section{AUTHORS PROFILE}
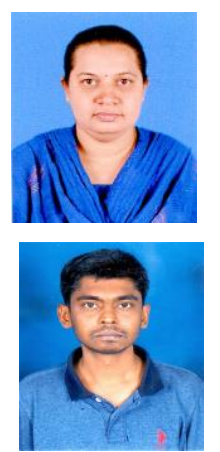

M.C Thriveni, Scientist-B at Central Sericultura Germplasm Resources Centre Education: M.Sc., Ph.D in Botany Published 22 research articles and one book chapter. Research area is Plant Taxonomy

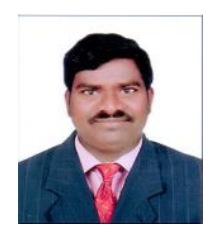

Raju Mondal, Scientist-B at Central Sericultural Germplasm Resources Centre

Education: M.Sc. in Botany; Pursuing Ph.D in BHU. Published 30 research articles. Research area: Plant Genetics

G. Thanavendan, Scientist- $C$ at Central Sericultural Germplasm Resources Centre.

Education: M.Sc., Ph.D. in Agricultural Entomolgy. Published 12 research articles. Research area: mulberry germplasm conservation and plant protection.

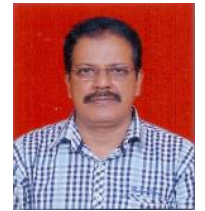

G. Ravikumar, Scientist \& Head of Biotechnology Division at Central Sericultural Germplasm Resources Centre.

Education: Ph.D. in Zoology from BHU. Published 34 research articles. Research area: Silkworm Biotechnology.



B.T. Sreenivasa, Director at Central Sericultural Germplasm Resources Centre. Education: Ph.D in Sericulture Published 11 research articles. Research area: mulberry germplasm conservation and plant protection

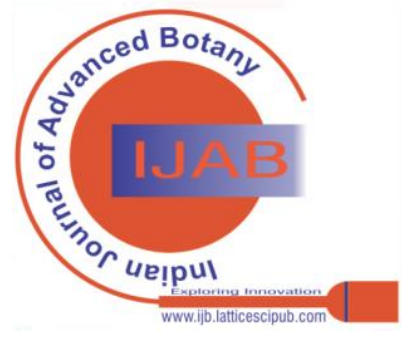

